\title{
Tunicamycin aggravates cell migration, cell invasion and cell proliferation in colonic epithelial cells
}

Rohit Gundamaraju ( $\nabla$ rohit.gundamaraju@utas.edu.au )

University of Tasmania Faculty of Health

\section{Ravichandra vemuri}

University of Tasmania

Ranga Rao Ambati

Vignan Group

Lakshminarayana Rangaswamy

Bangalore University

Wenying Lu

University of Tasmania

Rajaraman eri

University of Tasmania

\section{Short Report}

Keywords: Tunicamycin, ERS, Cell migration, Cell proliferation, Colonic epithelial cells

Posted Date: February 19th, 2021

DOI: https://doi.org/10.21203/rs.3.rs-212943/v1

License: (c) (i) This work is licensed under a Creative Commons Attribution 4.0 International License.

Read Full License 


\section{Abstract}

Aim: Endoplasmic reticular stress (ERS) has been well-documented in escalating metastasis in clinical conditions. There has been a debate on its role in cancer portraying it as a double-edged sword. However, there is no evidence of increased cell migration and cell invasion under increased ERS by tunicamycin(TUN) $10 \mu \mathrm{g} / \mathrm{mL}$.

Methods: In this study, we treated colonic epithelial cells (LS174T cells) with TUN and measured cell proliferation (by Ki67+ assay), cell migration and cell invasion at the $6^{\text {th }}$ hour specifically.

Results: Our results have demonstrated a positive correlation between TUN and cell migration, cell invasion, and proliferation. TUN treatment has significantly escalated cell invasion, migration and proliferation in LS174T cells.

Conclusion: These preliminary results clearly suggest that TUN might promote metastasis of gastrointestinal cancers.

\section{Introduction}

Chemotherapy can improve the quality of life and survival against various chronic diseases. Designing an anti-cancer drug requires specific pathways or receptors to target. Endoplasmic reticular stress (ERS) has been debated for its varied role in cancer. ERS triggers apoptosis to help cells adopt a harsh environment and on the other hand, ERS aids cells to evade cell death and gain survival ability through the up-regulation of adaptive measures[1]. Unfolded protein response (UPR) is an organized response program after the accumulation of misfolded proteins and stimulating stress in the ER[2]. In cancer, UPR is postulated to set the paradoxical environment and aid in cancer therapy resistance[3]. Further, ERS may trigger chronic inflammation in numerous immune and inflammatory disorders[4].

Cell migration allows cells to change their position between tissues or organs whereas; cell invasion is the ability of cancer cells to penetrate tissue barriers. Cell migration and invasion are collectively involved in cancer metastasis, which in turn cause cancer lethality. Therefore, it is obvious that the development of novel anti-cancer and anti-metastatic drugs can be made possible only upon knowledge of metastasis and their mediated mechanisms. In this regard, it is of utmost necessityto screen cell migration and invasion in cancer progression. Recently, the researchers Chang et al have indicated the role of heat shock protein Hspa5 in cell migration and invasion and correlated the influence of ERS in cancer [5].

On the other hand, glycosylation plays a huge role in the folding, stability, subcellular localization and biological functions of glycoproteins. Aberrant glycosylation has been identified as an essential hallmark of cancer and correlates with the development, progression, metastasis and chemoresistance of tumors. TUN, is a canonical compound for blocking N-linked glycosylation by inhibiting the transfer of UDP-Nacetylglucosamine (GIcNAc) to dolichol phosphate in the endoplasmic reticulum (ER) of eukaryotic cells, thus disrupting protein maturation [6]. Studies have reported that TUN induced cell death via mechanisms 
mediated by ROS and most essentially mTORC1 in prostate cancer cells lines [7]. However, recent evidence reports that (ER) stress is believed to contribute to several other steps along with metastasis and proliferation process of various types of cancers. For example, ER proteins such as XBP1, PERK, ATF6 and ATF4 that are involved in ER stress have been reported to participate in tumor growth and metastasis [8-10]. Further, there has been no study previously which has reported the time point mediated deleterious effects of ERS induced by TUN.

Previously, we reported that increased ERS triggers inflammation, impedes apoptosis and increases proliferation of cancer cells specifically at the 6th hour time point experiment[2]. Hence, in this study, we verified the role of tunicamycin (TUN) in promoting cell migration, invasion and proliferation in LS174T cells.

\section{Material And Methods}

\section{Cell culture}

The human colon cancer cell line (LS174T-ATCC $\AA$ CL-188 $8^{\text {TM }}$ ) were cultured in RPMI media with added Lglutamine (Life Technologies) supplemented with $10 \%$ fetal bovine serum, penicillin $(1000 \mathrm{UG} / \mathrm{mL}$ ) and streptomycin $(1000 \mathrm{U} / \mathrm{mL})$ (Gibco BRL, AUS). Cells were incubated under $37^{\circ} \mathrm{C}$ and $5 \% \mathrm{CO}_{2}$. After reaching confluence, the cells were harvested using $0.25 \%$ TrypLe express (Life Technologies, AUS). The detached cells were determined for cell number and viability after washing by using Countess $®$ cell counter (Life Technologies, AUS) as per the instructions.

\section{Cell invasion assay}

Cell invasion assay was performed in 24-well transwell chambers (Costar, Cambridge, MA, USA) containing polycarbonate filters with 8- $\mu \mathrm{m}$ pores coated with Matrige $^{\mathrm{Tm}}(1 \mathrm{mg} / \mathrm{mL}$, BD Sciences, San Jose, CA, USA). Briefly, $3 \times 10^{4}$ cells in $500 \mu \mathrm{L}$ of serum-free medium were seeded into the upper chamber, and the lower chamber was filled with $800 \mu \mathrm{L}$ of medium containing $10 \%$ FBS. After 24 -incubation, the non-invasive cells were removed with a cotton swab; the number of cells that had invaded through the basement membrane was counted. The cell numbers counted were averaged in six random fields at a magnification of $100 \mathrm{X}$.

\section{Cell migration assay}

Cell migration assay was performed using Boyden Chambers (Transwell Costar, 6.5-mm diameter, 8- $\mu \mathrm{m}$ pore size, Sigma, USA) according to the manufacturer's instructions. Briefly, $3 \times 10^{4}$ cells were resuspended in $500 \mu \mathrm{L}$ serum-free medium and seeded into the upper chamber and medium containing $(800 \mu \mathrm{L}) 10 \%$ FBS was added into the lower chamber. Cells were allowed to migrate for 24 hours and counted cell numbers under an inverted microscope. 
After incubation of cells with the respective treatments, the cultures were subjected to centrifugation (250xg for $4 \mathrm{~min}$ ) and collected supernatants for the determination of cytotoxicity by lactate dehydrogenase (LDH) assay. An aliquot of $50 \mu \mathrm{L}$ complete medium (blank), and cells (control), and cells treated with TUN supernatants obtained after the respective time point incubations were mixed with 100 $\mu \mathrm{L}$ of a solution containing the LDH assay mixture (LDH substrate, LDH dye, and LDH cofactor). The mixture was then incubated at room temperature for $30 \mathrm{~min}$, and the reaction was quenched by the addition of $1 \mathrm{~N}$ hydrochloric acid $(15 \mu \mathrm{L})$. The absorbance was measured at $490 \mathrm{~nm}$ using a microplate reader (Spectra Max M2, Sunnyvale, CA, USA). Cell viability was examined by a trypan blue dye exclusion staining using Countess Automated Cell Counter (Thermo-Fisher, Waltham, MA, USA).

\section{Cell proliferation assay (Ki67)}

Briefly, the treated cells were stained with Ki67+ and corresponding CY3-conjugated secondary antibody (Abcam, USA). The nucleus was counter stained with DAPI. The cells were inspected and documented the cell images using confocal microscopy (Olympus, Tokyo, Japanfv 1000).

\section{Statistical analysis}

Statistical significance of the differences between groups among repeated experiments was calculated by one-way and two-wayANOVA and the Fisher's LSD-test using GraphPad Prism 7 software (GraphPad Software Ltd, La Jolla, CA, USA). The results are expressed as the mean $(n=3)$ values \pm standard deviation. The difference in mean was considered significant at $p<0.05$.

\section{Results}

\section{TUN enhances cell migration and cell invasion in colon cancer cells}

The statistical graph of relative cell migration and invasion changes was shown as a fold increase relative to control after normalization to cell viability. We have assessed the migration ability of cells by transwell migration assay in LS174T cells upon treatment with TUN $(10 \mu \mathrm{g} / \mathrm{mL})$ for 6-hours. We have chosen a 6-hour incubation time period after successfully establishing the dynamics of ERS with Grp78 in our previous study [2], where we have noted maximum ERS at this time point. Our results suggest that an increase in ERS by TUN results in significant enhancement of cell migration as compared to nontreated cells (Figure $1 \mathrm{~B}$ ). Additionally, we performed a Matrigel invasion assay in these cells to investigate whether TUN treatment leads to increased invasion activity. Cells with TUN treatment have shown escalated cell invasion ability (Figure 1A). We have not recorded that cells were not prone to death recorded by the LDH assay (Figure 1C) which shows that the TUN treatment didn't kill the cells at the $6^{\text {th }}$ hour time point.

\section{TUN treatment leads to increased cell proliferation}


Considering our previous study [2], where ERS reduction lead to reduction in the proliferation of LS174T cells measured by EdU click assay, we employed Ki67 immunofluorescence (IF) assay to evaluate proliferation levels in LS174T cells. In untreated cells, we observed the least expression of Ki67 as compared to the cells treated with TUN10 $\mu \mathrm{g} / \mathrm{mL}$. TUN treatment led to an increase in the Ki67 expression, which demonstrates the potential proliferative ability of ERS in cancer.

\section{Discussion}

The present study imparts the preliminary evidence on the effects of ERS by TUN on carcinogenicity. The observation of this study indicated TUN increased the cancer invasion and migration significantly. This demonstrated the pro-carcinogenic effects of TUN on cancer cell migration, invasion and proliferation after successfully outlining the negative effects of ERS on cancer cell apoptosis and proliferation in our previous study [2].

Invasion, migration and proliferation have been described as hallmarks of cancer progression. TUN under in vitro conditions not only up-regulates ERS markers, but also promotes cell metastasis ability in thyroid cancer cells [11]. Some findings also suggest that TUN might escalate the metastatic ability via key chaperones like GRP78. We have previously observed surge in the levels of GRP78 in LS174T cells [2] and also in Winnie, a murine model of ER stress (Supplement Fig. 1-unpublished results).

In present study, an increase in Ki67 expression in LS174T cells upon treatment with TUN, which supports our previous study on cellular proliferation [2], was also observed. In a few conditions like pancreatic cancers, the tumor environment turns hypoxic where ER stress levels are high not only due to hypoxia, but likely due to loss of glucose. Cellular adaptation to this environment is certainly linked to the survival and proliferation of malignant cells. In pancreatic cancer cells, activation of the PERK and IRE1 arms of the UPR is delayed in the presence of ER stress compared to normal pancreatic cells. This was attributed to an abundance of protein folding machinery, such as chaperones. Additionally, once activated, the prosurvival XBP1 was noted to be activated for a longer time period in cancer cells when compared to normal cells[12].

Cell surface ERS proteins like HSPA5 have been found to enhance cell migration and invasion in colon cancer[13]. Post normalization of cell viability, blockade of surface HSPA5 significantly reduced cell migration and invasion [5]. ERS and UPR activation have been well correlated in the activation of numerous cancers [14]and ERS modulators were described as potential cancer therapeutics [15].

The involvement of ERS in cancer proliferation and metastasis has been well established with correlating to PI3K/AKT pathway. PI3K/AKT acts upstream of ER stress to affect fibroblast proliferation, resulting in bleomycin-induced pulmonary fibrosis in a previous study [16]. Hsu et al. demonstrated that the treatment with ER stress inhibitors or a PI3K inhibitor caused a successful reduction in fibroblast proliferation and improved pulmonary function [16]. Employment of angiotensin inhibitors, used to treat patients with high blood pressure, increased the survival of patients with metastatic renal cell carcinoma, reduced tumor fibrosis and enhanced chemotherapeutic efficacy in experimental models of cancer, possibly due to 
inhibiting growth factor-stimulated PI3K signaling [17]. These results also indicated that ERS suppression reduced cancer cell proliferation and metastasis.

Hence, after establishing a successful correlation between TUN and cell migration, invasion and proliferation from previous and current studies, we have inter-related the relation between ERS and cancer cell metastasis. This also helps to explore further potential ERS related cancer therapeutic targets.

\section{Conclusion}

In conclusion, the potential of UPR through TUN to regulate cell fate has been highlighted as a primary pathophysiology research focus through our study and represents a potential cancer therapeutic axis if we can modulate ERS by chemical or small molecule inhibitors.

\section{Declarations}

Author contributions: RG designed, performed the experiments and drafted the manuscript; RCV assisted in the experiments; RA and LR performed and analyzed partial experiments; WL assisted in experiments and in manuscript formatting; RE approved and checked the manuscript.

\section{Consent to participate: NA}

Consent to Publish: Approved by all the authors.

Funding: This work was supported by Takeda-IBD Research Grant (E0025316) allocated to Dr. Rajaraman Eri

Conflict of interest: We authors declare no conflict of interest.

\section{References}

1. Wang WA, Groenendyk J, Michalak M. Endoplasmic reticulum stress associated responses in cancer. Biochim Biophys Acta. (2014);1843:2143-9.

2. Gundamaraju R, Vemuri R, Chong WC, Myers S, Norouzi S, et al. Interplay between Endoplasmic Reticular Stress and Survivin in Colonic Epithelial Cells. Cells. (2018);7.

3. Yadav RK, Chae SW, Kim HR, Chae HJ. Endoplasmic reticulum stress and cancer. J Cancer Prev. (2014);19:75-88.

4. Hasnain SZ, Lourie R, Das I, Chen AC, McGuckin MA. The interplay between endoplasmic reticulum stress and inflammation. Immunol Cell Biol. (2012);90:260-70.

5. Chang YW, Chen HA, Tseng CF, Hong CC, Ma JT, et al. De-acetylation and degradation of HSPA5 is critical for E1A metastasis suppression in breast cancer cells. Oncotarget. (2014);5:10558-70.

6. Wu J, Chen S, Liu H, Zhang Z, Ni Z, et al. Tunicamycin specifically aggravates ER stress and overcomes chemoresistance in multidrug-resistant gastric cancer cells by inhibiting $\mathrm{N}$-glycosylation. 
J Exp Clin Cancer Res. (2018);37:272.

7. Guha P, Kaptan E, Gade P, Kalvakolanu DV, Ahmed H. Tunicamycin induced endoplasmic reticulum stress promotes apoptosis of prostate cancer cells by activating mTORC1. Oncotarget. (2017);8:68191-207.

8. Feng YX, Jin DX, Sokol ES, Reinhardt F, Miller DH, et al. Cancer-specific PERK signaling drives invasion and metastasis through CREB3L1. Nat Commun. (2017);8:1079.

9. Limia CM, Sauzay C, Urra H, Hetz C, Chevet E, et al. Emerging Roles of the Endoplasmic Reticulum Associated Unfolded Protein Response in Cancer Cell Migration and Invasion. Cancers (Basel). (2019);11.

10. Zhu H, Chen X, Chen B, Chen B, Song W, et al. Activating transcription factor 4 promotes esophageal squamous cell carcinoma invasion and metastasis in mice and is associated with poor prognosis in human patients. PLoS One. (2014);9:e103882.

11. Zhao G, Kang J, Xu G, Wei J, Wang X, et al. Tunicamycin promotes metastasis through upregulating endoplasmic reticulum stress induced GRP78 expression in thyroid carcinoma. Cell Biosci. (2020);10:115.

12. Riha R, Gupta-Saraf P, Bhanja P, Badkul S, Saha S. Stressed Out - Therapeutic Implications of ER Stress Related Cancer Research. Oncomedicine. (2017);2:156-67.

13. Li Z, Zhang L, Zhao Y, Li H, Xiao H, et al. Cell-surface GRP78 facilitates colorectal cancer cell migration and invasion. Int J Biochem Cell Biol. (2013);45:987-94.

14. Wang M, Kaufman RJ. The impact of the endoplasmic reticulum protein-folding environment on cancer development. Nat Rev Cancer. (2014);14:581-97.

15. Farooqi AA, Li KT, Fayyaz S, Chang YT, Ismail M, et al. Anticancer drugs for the modulation of endoplasmic reticulum stress and oxidative stress. Tumour Biol. (2015);36:5743-52.

16. Hsu HS, Liu CC, Lin JH, Hsu TW, Hsu JW, et al. Involvement of ER stress, PI3K/AKT activation, and lung fibroblast proliferation in bleomycin-induced pulmonary fibrosis. Sci Rep. (2017);7:14272.

17. Kai F, Laklai H, Weaver VM. Force Matters: Biomechanical Regulation of Cell Invasion and Migration in Disease. Trends Cell Biol. (2016);26:486-97.

\section{Figures}




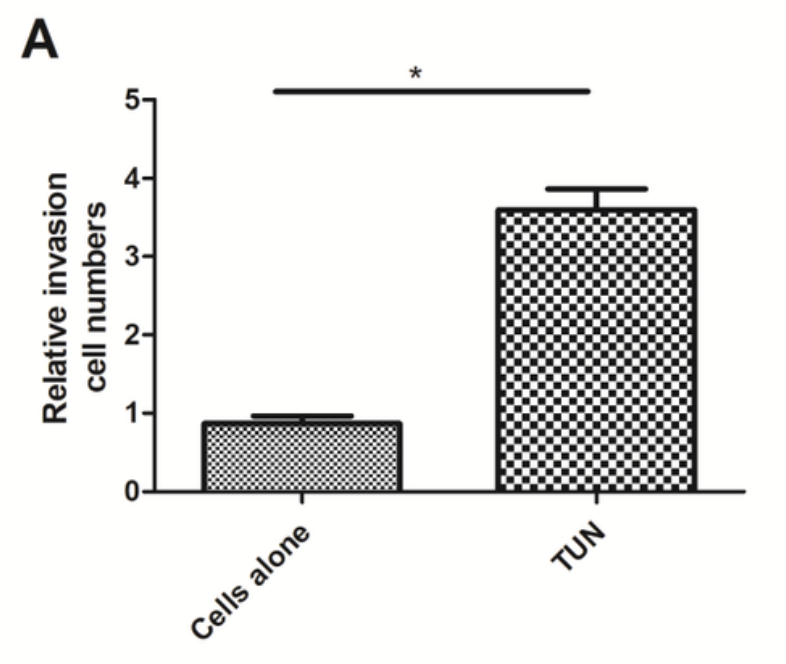

B
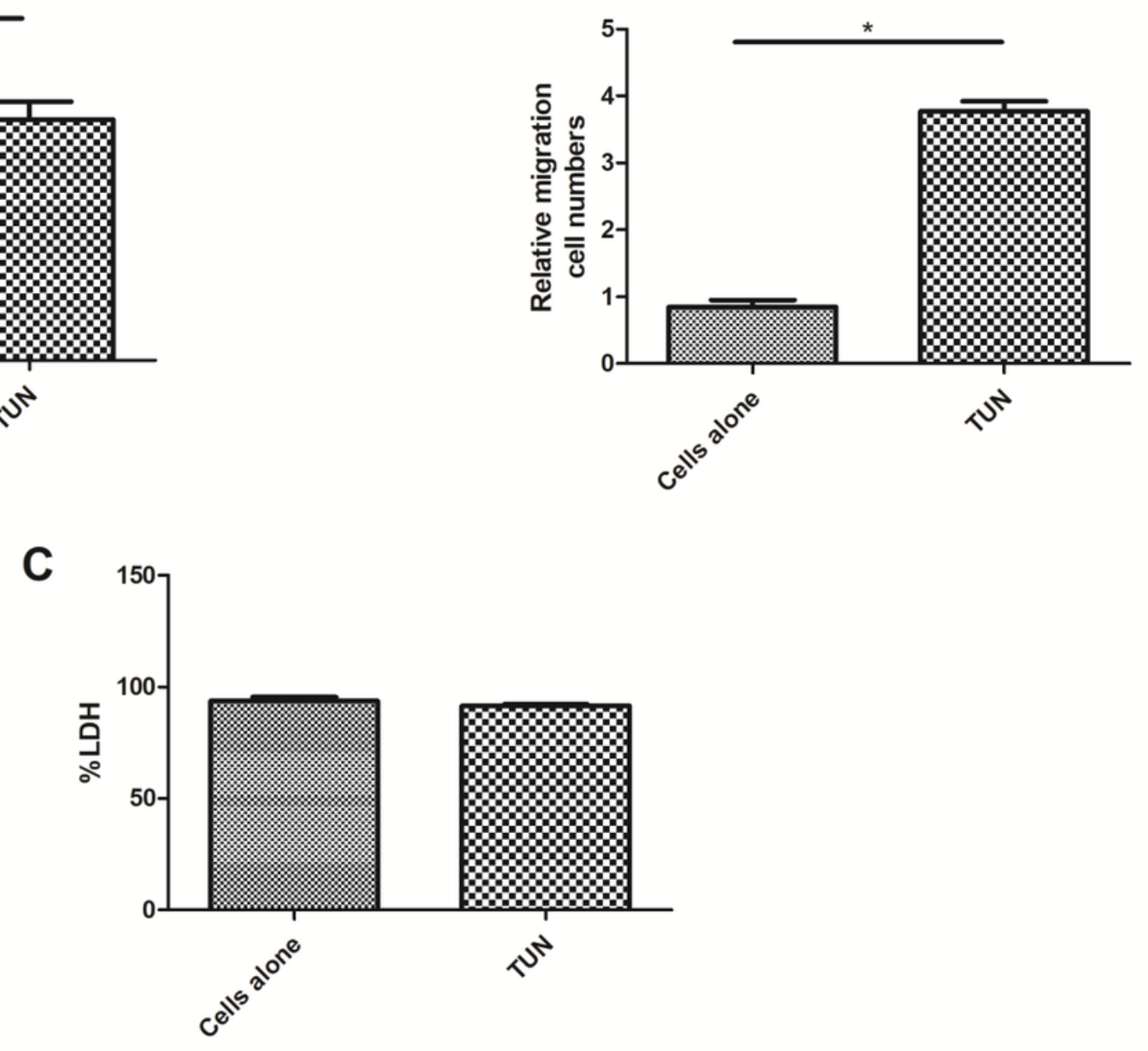

\section{Figure 1}

Effects of TUN on cell migration and cell invasion. A) Cell invasion assay of the cells alone and cells induced with $10 \mu \mathrm{g} / \mathrm{mL}$ of TUNB) Cell migration assay of the cells alone and cells induced with $10 \mu \mathrm{g} / \mathrm{mL}$ of TUNC) Lactose dehydrogenase assay of the cells alone and cells induced with $10 \mu \mathrm{g} / \mathrm{mL}$ of TUN showcasing cell death. 

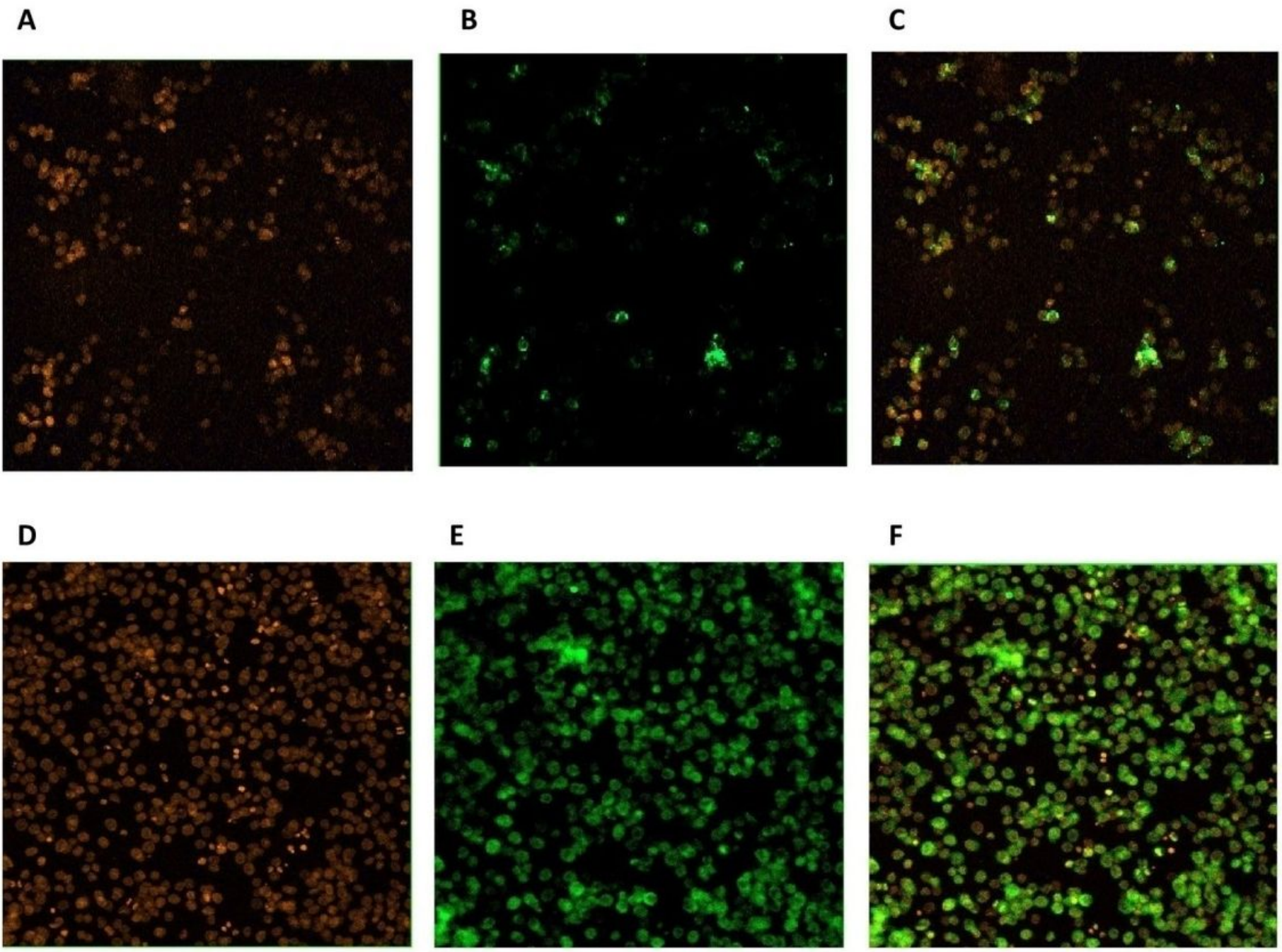

Figure 2

Ki67 assay of cells upon treatment with TUN10 $\mu \mathrm{g} / \mathrm{mL}$. A) DAPI staining of non-treated cells B) Ki67+ of non-treated cells C) Merge D) DAPI staining of cells treated with $10 \mu \mathrm{g} / \mathrm{mL}$ TUN E) Ki67+ of cells treated with $10 \mu \mathrm{g} / \mathrm{mL}$ TUN F) Merge 